Low frequency azimuthal stability of the ionization region of the Hall thruster discharge. II. Global analysis

D. Escobar' and E. Ahedo'

Citation: Phys. Plasmas 22, 102114 (2015); doi: 10.1063/1.4934352

View online: http://dx.doi.org/10.1063/1.4934352

View Table of Contents: http://aip.scitation.org/toc/php/22/10

Published by the American Institute of Physics 


\title{
Low frequency azimuthal stability of the ionization region of the Hall thruster discharge. II. Global analysis
}

\author{
D. Escobar ${ }^{1, a)}$ and E. Ahedo ${ }^{2, b)}$ \\ ${ }^{1}$ Universidad Politécnica de Madrid, 28040 Madrid, Spain \\ ${ }^{2}$ Universidad Carlos III de Madrid, 28911 Leganés, Spain
}

(Received 2 June 2015; accepted 7 October 2015; published online 21 October 2015)

The linear stability of the Hall thruster discharge is analysed against axial-azimuthal perturbations in the low frequency range using a time-dependent 2D code of the discharge. This azimuthal stability analysis is spatially global, as opposed to the more common local stability analyses, already afforded previously (D. Escobar and E. Ahedo, Phys. Plasmas 21(4), 043505 (2014)). The study covers both axial and axial-azimuthal oscillations, known as breathing mode and spoke, respectively. The influence on the spoke instability of different operation parameters such as discharge voltage, mass flow, and thruster size is assessed by means of different parametric variations and compared against experimental results. Additionally, simplified models are used to unveil and characterize the mechanisms driving the spoke. The results indicate that the spoke is linked to azimuthal oscillations of the ionization process and to the Bohm condition in the transition to the anode sheath. Finally, results obtained from local and global stability analyses are compared in order to explain the discrepancies between both methods. (C) 2015 AIP Publishing LLC.

[http://dx.doi.org/10.1063/1.4934352]

\section{INTRODUCTION}

During the last few years, a growing interest in the so-called azimuthal spoke ${ }^{2}$ oscillation has reappeared within the Hall Effect Thruster community. Even though the presence of this low frequency $(5-30 \mathrm{kHz})$ oscillation has been known for a long time, ${ }^{3-5}$ the interest has been renewed recently thanks to various experimental results presented in different series of publications by Raitses et al., ${ }^{6-11}$ McDonald et al., ${ }^{12-17}$ and Liu et al. ${ }^{18,19}$ The spoke normally appears as a long wavelength low frequency oscillation travelling in the $\mathbf{E} \times \mathbf{B}$ direction with an azimuthal phase speed of a few $\mathrm{km} / \mathrm{s}$. This oscillation is a relevant feature of the Hall discharge physics and may play an important role in various aspects of the Hall discharge such as electron conductivity or incomplete ionization. A review of experimental and theoretical analyses of the spoke is presented in Part I of this study ${ }^{1}$ and thus is omitted here.

This paper analyses the low frequency azimuthal stability of the Hall discharge globally, contrary to the more common local stability studies. The latter have been widely used in the literature to analyze the azimuthal stability of the Hall discharge. 1,5,20-29 However, these local methods require freezing the steady-state background solution at each axial location and reduce the perturbation analysis to local normal modes. For this to be consistent, the wavelength of the oscillations must be much smaller than the length scale of variation of the macroscopic variables. Given the large spatial gradients of the Hall discharge, this condition is not strictly met in Hall thrusters. However, global linear methods

\footnotetext{
${ }^{\text {a)} P h . D . ~ C a n d i d a t e, ~ E q u i p o ~ d e ~ P r o p u l s i o ́ n ~ E s p a c i a l ~ y ~ P l a s m a s ; ~ U R L: ~ h t t p: / / ~}$ aero.uc 3 m.es/ep2.

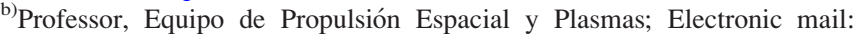
eduardo.ahedo@uc3m.es.
}

consider consistently the axial variation of the macroscopic variables along the thruster. Global methods have indeed been used in the past to analyze the stability of Hall thrusters in the high-frequency range. ${ }^{30-33}$ In order to carry out the global analysis, the $1 \mathrm{D}$ model of Ahedo et al. ${ }^{34}$ including heat conduction and wall losses is used as starting point. This 1D model has been used thoroughly in the past to analyze different aspects of the Hall discharge. ${ }^{35-41}$

The rest of the paper is organized as follows. In Section II, the formulation of the linearised time-dependent two-dimensional (2D) model used is summarized. Section III discusses the results of the linear model for the breathing mode and the spoke. Simplified models are used in Section IV to better understand the detected oscillations. Section V presents a comparison between local and global methods. Finally, Section VI is devoted to conclusions.

\section{LINEAR TIME-DEPENDENT 2D MODEL}

This section summarizes the formulation of the linear time-dependent 2D model used here, which is similar to that of Part I. ${ }^{1}$ The formulation is based on the 1D model of Ahedo et al., ${ }^{34,35}$ which has been widely used in the past for modelling the plasma in Hall thrusters. That 1D model can be extended to two dimensions, azimuthal $(y)$ and axial $(x)$, with time-dependent terms. Under the same hypotheses of the $1 \mathrm{D}$ model, the governing 2D time-dependent equations for singly charged ions, electrons, and neutrals may be written as

$$
\begin{gathered}
\frac{\partial n}{\partial t}+\frac{1}{d_{c}} \nabla \cdot\left(n \mathbf{v}_{e} d_{c}\right)=\frac{\partial n}{\partial t}+\frac{1}{d_{c}} \nabla \cdot\left(n \mathbf{v}_{i} d_{c}\right) \\
=-\frac{\partial n_{n}}{\partial t}-\frac{1}{d_{c}} \nabla \cdot\left(n_{n} \mathbf{v}_{n} d_{c}\right)=n\left(\nu_{i}-\nu_{w}\right),
\end{gathered}
$$




$$
\begin{gathered}
m_{i}\left(\frac{\partial}{\partial t}\left(n_{n} \mathbf{v}_{n}\right)+\frac{1}{d_{c}} \nabla \cdot\left(n_{n} \mathbf{v}_{n} \mathbf{v}_{n} d_{c}\right)\right) \\
=-m_{i} n\left(\nu_{i} \mathbf{v}_{n}-\nu_{w} \mathbf{v}_{n w}\right), \\
m_{i}\left(\frac{\partial}{\partial t}\left(n \mathbf{v}_{i}\right)+\frac{1}{d_{c}} \nabla \cdot\left(n \mathbf{v}_{i} \mathbf{v}_{i} d_{c}\right)\right) \\
=-e n \nabla \phi+m_{i} n\left(\nu_{i} \mathbf{v}_{n}-\nu_{w} \mathbf{v}_{i}\right), \\
0=-\nabla\left(n T_{e}\right)-e n\left(-\nabla \phi+\mathbf{v}_{e} \times \mathbf{B}\right)-m_{e} n \nu_{e} \mathbf{v}_{e}, \\
\frac{\partial}{\partial t}\left(\frac{3}{2} n T_{e}\right)+\frac{1}{d_{c}} \nabla \cdot\left(\frac{5}{2} n T_{e} \mathbf{v}_{e} d_{c}+\mathbf{q}_{e} d_{c}\right) \\
=e n \mathbf{v}_{e} \cdot \nabla \phi-n \nu_{i} E_{i}^{\prime}-n \nu_{w e} T_{e}, \\
0=\frac{5}{2} n T_{e} \nabla T_{e}+e \mathbf{q}_{e} \times \mathbf{B}+m_{e} \nu_{e} \mathbf{q}_{e},
\end{gathered}
$$

where $e, m_{e}$, and $m_{i}$ are the electron charge, electron mass, and ion mass, respectively; $\mathbf{E}$ and $\mathbf{B}$ are the electric and magnetic fields; $n_{n}$ and $n$ are neutral and plasma particle densities; $\mathbf{v}_{n}, \mathbf{v}_{e}$, and $\mathbf{v}_{i}$ are fluid velocities of neutrals, electrons, and ions, respectively; $T_{e}, \phi$, and $\mathbf{q}_{e}$ are the electron temperature, electric potential, and electron heat flux, respectively; $\nu_{e}$ is the effective electron collision frequency, $\nu_{e}=\nu_{B}$ $+\nu_{w m}+\nu_{e n}+\nu_{e i}$, accounting for Bohm-type diffusion, $\nu_{B}$, near-wall conductivity, $\nu_{w m}$, electron-neutral collisions, $\nu_{e n}$, and electron-ion collisions, $\nu_{e i} ; \nu_{i}, \nu_{w}$, and $\nu_{w e}$ represent the frequencies for ionization, particle wall-recombination, and energy losses at lateral walls, respectively; $E_{i}^{\prime}$ models the energy loss per ion-electron due to ionization and radiation; $d_{c}(x)$ is the plasma beam width, constant within the channel and increasing outside due to plume divergence. The expression used for Bohm-diffusion is $\nu_{B}=\alpha_{B} \omega_{c e}$, where $\omega_{c e}=e B /$ $m_{e}$ is the electron cyclotron frequency and $\alpha_{B}$ is a constant empirical coefficient whose value is typically selected so as to match approximately experimental results $\left(\alpha_{B} \sim 0.01\right)$. Ions impacting the chamber walls undergo wall recombination and return to the channel as neutrals. The velocity of the newly born neutrals, $\mathbf{v}_{n w}$, is not equal to the velocity of impacting ions but is the result of an accommodation process in the wall, $\mathbf{v}_{n w}=a_{w} \mathbf{v}_{n}+\left(1-a_{w}\right) \mathbf{v}_{i}$, where $a_{w}$ is the accommodation factor. The no-accommodation limit corresponds to the case $a_{w}=0$. Expressions for all terms of this formulation may be found elsewhere. ${ }^{34,35}$

The radial variation of the macroscopic variables is neglected here, because of their presumable negligible role on azimuthal-axial oscillations, thus reducing the problem to twodimensions: axial and azimuthal. Moreover, curvature effects in the azimuthal direction are also neglected as the mean radius of the channel, $R_{m}$, is typically larger than its width. In the limit of a stationary and axi-symmetric solution, the system of equations (1) reduces to the 1D model. Note that the model given by Eqs. (1) includes both heat conduction terms and plume divergence as well as Bohm-type diffusion and losses to the lateral walls. Plume divergence and lateral losses were not included in the formulation used in Part I of this study. ${ }^{1}$

The small perturbation hypothesis allows linearising the model in Eqs. (1) around a given background axi-symmetric, stationary solution. The perturbations may then be assumed to be of the following Fourier form:

$$
\hat{f}(t, x, y)=\bar{f}(x) \exp \left(-i \omega t+i k_{y} y\right),
$$

where $\bar{f}(x)$ is the $x$-dependent coefficient of the perturbation of a generic variable $f$, and $\omega$ and $k_{y}$ are the angular frequency and the azimuthal wave number, respectively. The wave mode number, $m$, is given by $m=-R_{m} k_{y}$ and can only have integer values due to azimuthal symmetry. The details of the method used to linearise Eqs. (1), split the zero-th and first order problems, solve the resulting system of equations, and find self-excited oscillations in the complex frequency space $\left(\omega=\omega_{r}+i \omega_{i}\right)$, have already been presented in the literature. ${ }^{42} \mathrm{~A}$ set of boundary conditions are necessary for the first order problem at anode, cathode, and internal sonic point. These boundary conditions are also obtained linearising the boundary conditions of the zero-th order problem. ${ }^{42}$

\section{RESULTS AND DISCUSSION}

\section{A. Background solutions}

The zero-th order solutions whose stability is to be analysed in Sections III B and III C are described here. For this purpose, a SPT-100 thruster model has been considered as a reference case.

The main simulation parameters are presented in Table I, where the following symbols are used: $\dot{m}$ is the mass flow rate through the anode; $V_{d}$ is the discharge voltage; $B_{\max }$ is the maximum magnetic field; $x_{\max }$ is the location of the maximum magnetic field with respect to the anode; $L_{A E}$ is the distance from anode to external cathode (i.e., neutralizing surface); $L_{c h}, d_{c}$, and $R_{m}$ are, respectively, the length, width, and mean radius of the channel; $T_{e E}$ is the cathode temperature; $v_{n B}$ is the neutral velocity at injection; $\tilde{\nu}_{w}$ is a dimensionless parameter of the wall losses model $;{ }^{34} T_{S E E}$ is the electron temperature yielding $100 \%$ of secondary electron emission for the specific wall material used; and $L_{m 1}$ and $L_{m 2}$ are inward and outward length scales characterizing the magnetic field profile. ${ }^{34}$ The magnetic field profile, $B(x)$, is an input of the simulation defined by $B_{\max }, L_{m 1}$, and $L_{m 2}$. The electron temperature at the anode, $T_{e B}$, and the discharge current, $I_{d}$, are outputs of the simulation. In the reference case defined above, these result in $T_{e B}=2.9 \mathrm{eV}$ and $I_{d}=5.4 \mathrm{~A}$.

Fig. 1 shows the axial profiles of the main macroscopic variables corresponding to the background solution for the reference case shown in Table I. The main differences with respect to previously reported solutions without heat conduction $^{43}$ are the following. First, no steep gradients of the temperature exist and the maximum temperature is

TABLE I. Main simulation parameters of the SPT-100 Hall Thruster used as reference case for the simulations.

\begin{tabular}{lccc}
\hline \hline$\dot{m}$ & $4.75 \mathrm{mg} / \mathrm{s}$ & $V_{d}$ & $300 \mathrm{~V}$ \\
$B_{\max }$ & $230 \mathrm{G}$ & $x_{\max }$ & $25 \mathrm{~mm}$ \\
$L_{A E}$ & $33.5 \mathrm{~mm}$ & $L_{c h}$ & $25 \mathrm{~mm}$ \\
$d_{c}$ & $15 \mathrm{~mm}$ & $R_{m}$ & $42.5 \mathrm{~mm}$ \\
$T_{e E}$ & $5 \mathrm{eV}$ & $v_{n B}$ & $300 \mathrm{~m} / \mathrm{s}$ \\
$\alpha_{B}$ & 0.0094 & $\tilde{\nu}_{w}$ & 0.17 \\
$T_{S E E}$ & $30 \mathrm{eV}$ & $a_{w}$ & 0.85 \\
$L_{m 1}$ & $15 \mathrm{~mm}$ & $L_{m 2}$ & $5 \mathrm{~mm}$ \\
\hline \hline
\end{tabular}



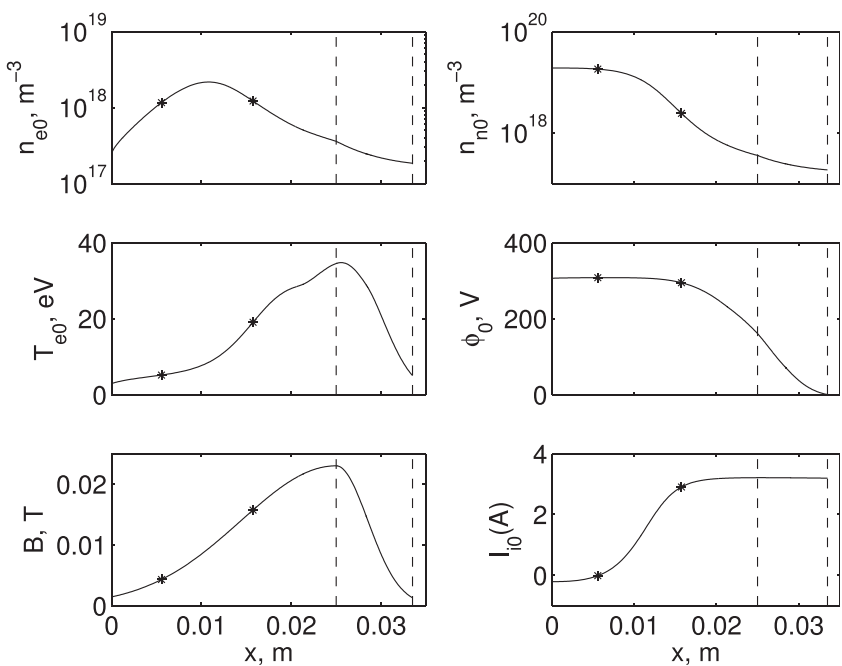

FIG. 1. Axial profiles of the main macroscopic variables of the background solution for the reference case: $x$ is the axial location, $n_{e 0}(x)$ is the plasma density, $n_{n 0}(x)$ is the neutral density, $T_{e 0}(x)$ is the electron temperature, $\phi_{0}(x)$ is the electric potential, $B(x)$ is the magnetic field, $I_{i 0}(x)$ is the ion axial current. The left asterisk corresponds to the zero-ion-velocity point, I, whereas the right asterisk corresponds to the regular ion sonic transition point inside the channel, $\mathrm{S}$. The space between both points corresponds roughly to the ionization region of the thruster. The left vertical dashed line represents the channel exit, whereas the right one indicates the location of the cathode, which is the end of the simulation domain.

smaller due to heat conduction and lateral wall losses. Second, there is a plateau in the temperature profile around the $30 \mathrm{eV}$ level as the saturation temperature of the wall losses model is reached. Third, the temperature peak is closer to the channel exit due to the selected magnetic field profile, whose maximum is near the channel exit plane. And last, the ionization region is closer to the anode as higher temperatures are reached inside the channel.

In order to analyze the influence of various operation parameters on azimuthal oscillations, it is interesting to perform variations on different parameters and analyze the stability of some limiting background solutions of those variations. As part of this analysis, the following parameters are varied: discharge potential, $V_{d}$; mass flow rate, $\dot{m}$; and channel width, $d_{c}$. These parametric variations have been previously performed and analyzed by the authors. ${ }^{37}$ Here, they are used to evaluate their possible influence on the axial and azimuthal oscillations. Table II shows the parameters used for the various background solutions whose stability is analysed in Sections III B and III C. The value of the parameters not shown in Table II is identical to those shown in Table I. Each background state is given an identifier for easier reference.

The following comments are worth about the trends observed in those parametric variations ${ }^{37}$ as they help understand the remainder of this study. As the discharge voltage is increased, the magnetic field typically needs to be varied roughly as $B_{\max } \propto V_{d}^{p}$, where $p \approx 0.5$, for optimum operation. At the same time, the overall efficiency ${ }^{37}$ of the thruster increases, wall losses and the maximum temperature scale linearly with the discharge voltage, and the ionization region moves upstream as shown previously. ${ }^{37}$ However, as the mass flow rate is increased, the neutral density and ionization frequency are increased, reducing the axial thickness of the
TABLE II. Frequency, $f(\mathrm{kHz})$, of different oscillation modes for several operation parameters. REF refers to the reference solution given in Fig. 1, and I, II, and III refer to the parametric variations on $V_{d}, \dot{m}$, and $d_{c}$, respectively. "-" indicates that no self-mode exists to the linear stability problem, $(+)$ indicates a stable solution, and (*) indicates an unstable solution referenced in the text below.

\begin{tabular}{|c|c|c|c|c|c|c|c|}
\hline \multirow[b]{2}{*}{ Case } & \multirow{2}{*}{$\frac{\text { Parameter }}{V_{d}, \mathrm{~V}}$} & \multirow{2}{*}{$\frac{\text { Parameter }}{B_{\text {max }}, \mathrm{G}}$} & \multirow{2}{*}{$\frac{m=0}{f, \mathrm{kHz}}$} & \multirow{2}{*}{$\frac{m=1}{f, \mathrm{kHz}}$} & \multirow{2}{*}{$\frac{m=2}{f, \mathrm{kHz}}$} & \multirow{2}{*}{$\frac{m=3}{f, \mathrm{kHz}}$} & \multirow{2}{*}{$\frac{m=4}{f, \mathrm{kHz}}$} \\
\hline & & & & & & & \\
\hline Ia & 20 & 23 & $11.1(+)$ & - & - & 22.2 & - \\
\hline $\mathrm{Ib}$ & 200 & 179 & $14.3(+)$ & 18.1 & 45 & 87.2 & - \\
\hline REF & 300 & 230 & 15.6 & 24.9 & 55.7 & 102 & - \\
\hline Ic & 500 & 313 & 16.9 & 39.8 & 119 & 24.8 & - \\
\hline Id & 700 & 383 & 17.9 & 53.5 & - & - & - \\
\hline Case & $\dot{m}, \mathrm{mg} / \mathrm{s}$ & $B_{\max }, \mathrm{G}$ & $f, \mathrm{kHz}$ & $f, \mathrm{kHz}$ & $f, \mathrm{kHz}$ & $f, \mathrm{kHz}$ & $F, \mathrm{kHz}$ \\
\hline IIa & 3.3 & 225 & 17.0 & 50.3 & 137 & - & - \\
\hline $\mathrm{IIb}$ & 4 & 226 & 16.3 & 30.3 & 84 & - & - \\
\hline $\mathrm{REF}$ & 4.8 & 230 & 15.6 & 24.9 & 55.7 & 102 & - \\
\hline IIc & 7 & 241 & 13.5 & 13.8 & 14.3 & 54.7 & - \\
\hline IId & 8.5 & 249 & 12.7 & $38.9(*)$ & 15.1 & - & - \\
\hline IIe & 10 & 256 & 12.1 & $27.2(*)$ & 11.1 & 8.9 & - \\
\hline Case & $d_{c}, \mathrm{~mm}$ & $B_{\max }, \mathrm{G}$ & $f, \mathrm{kHz}$ & $f, \mathrm{kHz}$ & $f, \mathrm{kHz}$ & $f, \mathrm{kHz}$ & $f, \mathrm{kHz}$ \\
\hline $\mathrm{REF}$ & 15 & 230 & 15.6 & 24.9 & 55.7 & 102 & - \\
\hline IIIa & 18 & 244 & 15.3 & 24.1 & 65.2 & 109 & 12.7 \\
\hline IIIb & 21 & 251 & 15.2 & 23.1 & 69.3 & 110 & 11.7 \\
\hline IIIc & 31 & 278 & 14.7 & 22.9 & 70.9 & 118 & 8.3 \\
\hline
\end{tabular}

ionization region, moving it downstream and increasing the efficiency of the thruster. Moreover, the length of the acceleration region is decreased while the temperature gradients are larger when the mass flow rate increases.

\section{B. Axial oscillations-Breathing mode}

This section is devoted to the analysis of low frequency axial oscillations, which are usually known as the breathing mode in the Hall thruster literature, using the linear timedependent 2D model and the background solutions presented above. The analysis focuses on the stability of the reference background solution and of some limiting solutions of the parametric variations. Similar analyses have been carried out in the past by other authors. ${ }^{39-41}$ However, the intention here is to show how the linear time-dependent $2 \mathrm{D}$ model presented above is capable of reproducing the breathing mode. ${ }^{2}$ Note that as the size of the perturbations does not result from the linear stability analysis, it has been set artificially in each case to illustrate visually the main properties of the oscillations. Moreover, the exponential time dependence of the linear perturbations, $\exp \left(-i \omega_{i} t\right)$, has been omitted from the figures for clarity as well.

Fig. 2 shows the contour maps in the $x-t$ space of the main variables of a purely axial $(m=0)$ unstable oscillation, for the reference case presented in Fig. 1 . The growth rate of the unstable oscillation is $\omega_{i} / 2 \pi \approx 0.5 \mathrm{kHz}$, whereas the frequency is $f=15.6 \mathrm{kHz}$, of the same order of magnitude as the values observed experimentally. ${ }^{2}$ The time evolution of the variables shown in Fig. 2 is qualitatively similar to those obtained with non-linear models of the breathing 

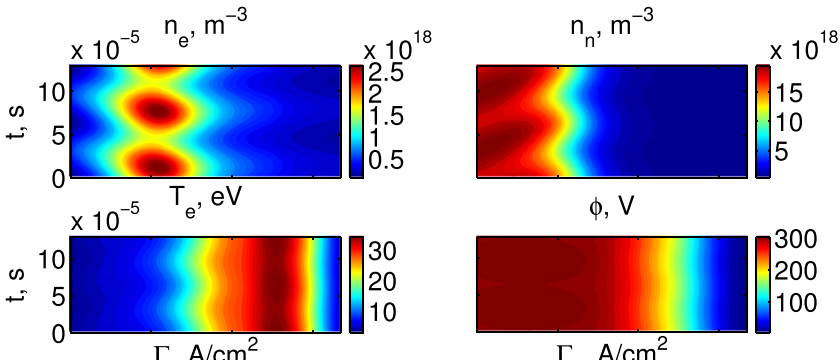

$\phi, \mathrm{V}$

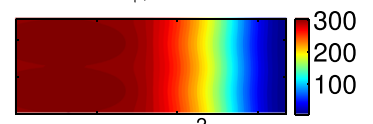

$\Gamma_{e}, \mathrm{~A} / \mathrm{cm}^{2}$
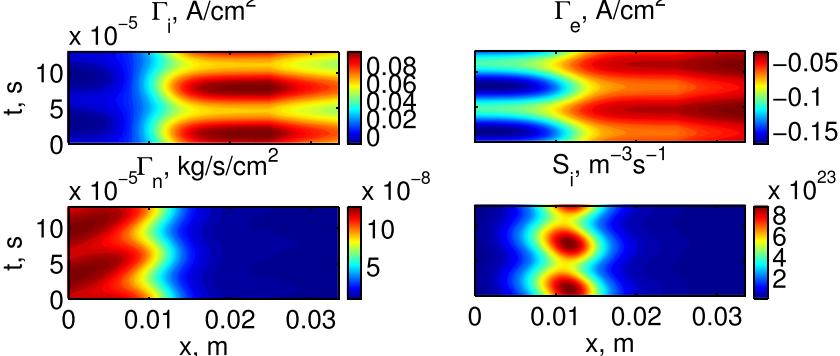

FIG. 2. Oscillations of the main macroscopic variables as combinations of the background solution and the perturbations shown as a function of $x$ and $t$ for the breathing mode $(m=0)$. The conditions are similar to those shown in Fig. 1. The variables shown are from left to right and from top to down: plasma density, $n_{e}$; neutral density, $n_{n}$; electron temperature, $T_{e}$; electric potential, $\phi$; ion axial flux, $\Gamma_{i}$; electron axial flux, $\Gamma_{e}$; neutral axial flux, $\Gamma_{n}$; and ionization source term, $S_{i}=n \nu_{i}$.

mode. ${ }^{41,44,45}$ Those figures reproduce the usual properties of the breathing mode, ${ }^{46}$ namely, (a) the ionization region moves back and forth as a consequence of the depletion and replenishment of neutrals; (b) a standing wave of plasma density exists in the rear part of the thruster in phase with the discharge current oscillation and with similar relative size; (c) a travelling wave, in combination with a standing wave, of neutral density is visible with a quarter-cycle phase difference with respect to the discharge current oscillation; and (d) the relative size of neutral oscillations is much smaller than those of plasma density and discharge current.

Table II shows the variation of the oscillation frequency of the simulated breathing mode with the discharge voltage and the mass flow. As the discharge voltage is increased or the mass flow decreased, the ionization region moves upstream ${ }^{37}$ in the background solution, and this could cause the oscillation frequency increase observed in the results.

\section{Axial-azimuthal oscillations-Spoke}

This section is devoted to the analysis of low frequency azimuthal oscillations with the help of the linear timedependent 2D model previously described.

\section{Reference solution}

The results of the low frequency global stability analysis in the azimuthal direction for the reference background solution are presented in this paragraph. Figs. 3-5 show an unstable $m=1$ azimuthal oscillation for the reference case shown in Fig. 1. This oscillation has the following properties: frequency $f=24.9 \mathrm{kHz}$ and azimuthal phase speed $v_{y}=6.6 \mathrm{~km} / \mathrm{s}$ in the $+\mathbf{E} \times \mathbf{B}$ direction. This numerical result is in
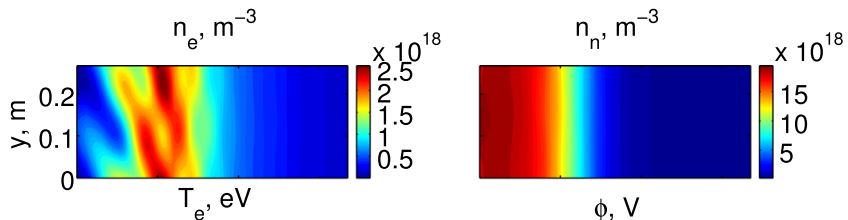

$\phi, \mathrm{V}$
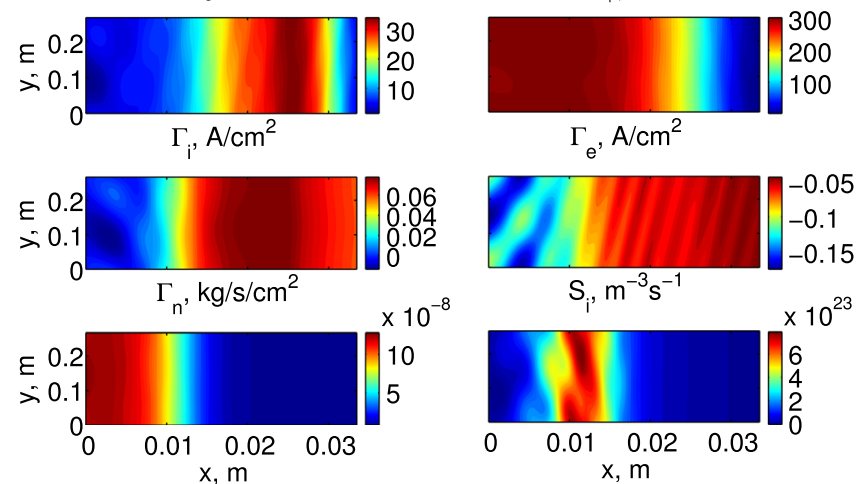

FIG. 3. Oscillations of the main macroscopic variables as combinations of the background solution and the perturbations shown as functions of $x$ and $y$ at $t=0$ for a $m=1$ self-excited oscillation of the perturbation problem for the reference case presented in Fig. 1. Variables shown (from left to right and upper to lower): plasma density, $n_{e}$; neutral density, $n_{n}$; electron temperature, $T_{e}$; electric potential, $\phi$; ion axial flux, $\Gamma_{i}$; electron axial flux, $\Gamma_{e}$; neutral axial flux, $\Gamma_{n}$; and ionization source term, $S_{i}=n \nu_{i}$.

qualitative agreement with experimental evidence, where it is normally observed that the spoke travels in the $+\mathbf{E} \times \mathbf{B}$ direction with a phase speed of several $\mathrm{km} / \mathrm{s}$ and a frequency higher than the breathing mode for the same thruster. ${ }^{2}$ In Figs. 3 and 4, it is possible to observe, respectively, contour maps in the $x-y$ space and the evolution in the $x-t$ space of the main macroscopic variables. Fig. 5 shows contour maps in the $x-y$ space for different instants of time, $t$, during one cycle of the azimuthal oscillation for plasma and neutral density. Some comments are worth relative to the solution shown in Figs. 3-5. First, the oscillation is mostly concentrated in
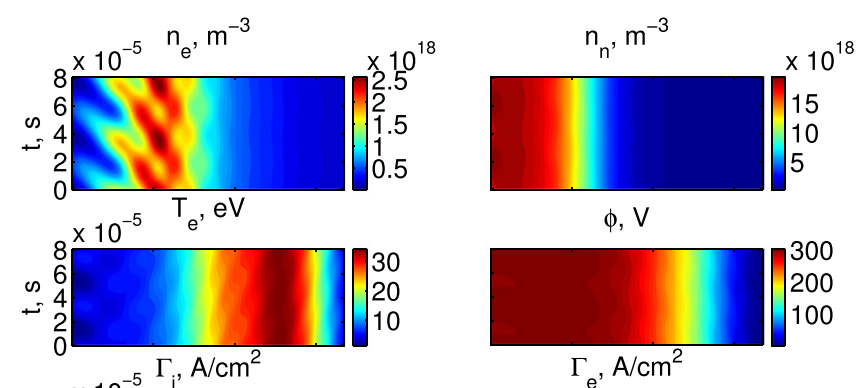

$$
\phi, \mathrm{V}
$$

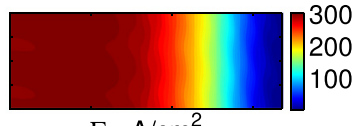

$\Gamma_{e}, \mathrm{~A} / \mathrm{cm}^{2}$
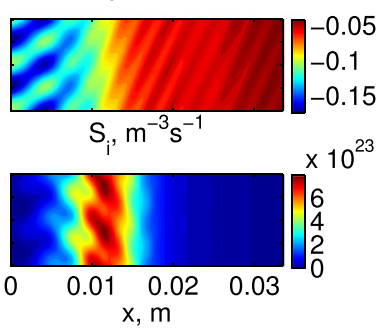

FIG. 4. Oscillations of the main macroscopic variables as combinations of the background solution and the perturbations shown as functions of $x$ and $t$ at $y=0$ for an $m=1$ self-excited oscillation of the perturbation problem for the reference case presented in Fig. 1. The variables shown are the same of Fig. 3. 

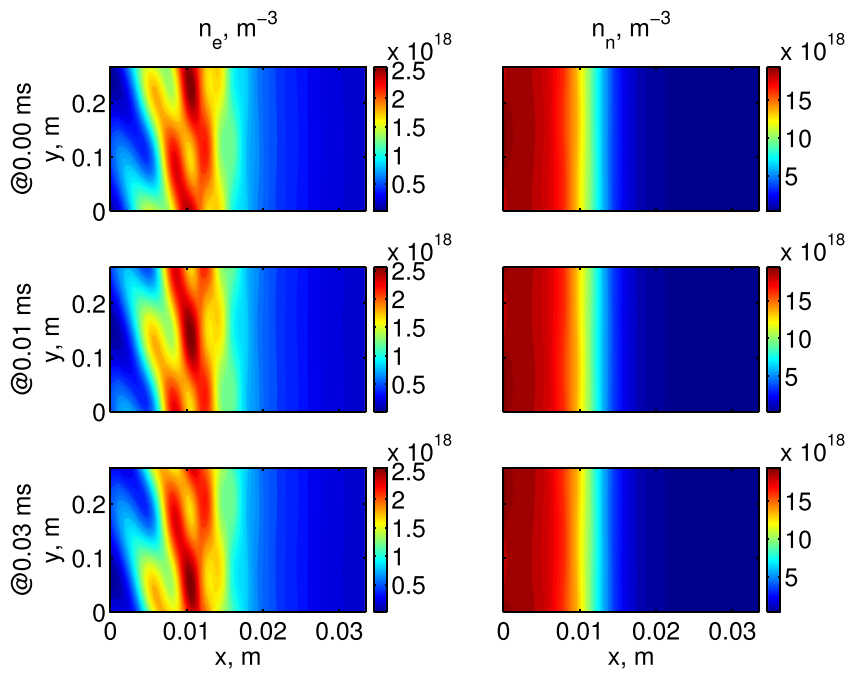

FIG. 5. Oscillations of the main macroscopic variables as combinations of the background solution and the perturbations shown as functions of $x$ and $y$ at different values of $t$ (at $\mathrm{t} \mathrm{ms}$ ) for the same conditions used previously in Fig. 4. Variables shown (from left to right): plasma density, $n_{e}$; neutral density, $n_{n}$.

the rear part of the thruster, upstream of the acceleration region, although there are some perturbations in temperature and electric field reaching the plume. And second, even if the relative size of the perturbation of the neutral density is negligible, the ionization front moves back and forth as in the case of the breathing mode. This motion of the ionization front seems linked to the plasma density oscillation as the temperature oscillation has lower relative size than that of plasma density.

Previous preliminary results obtained in other studies by the authors ${ }^{43}$ with a model without heat conduction also show low frequency azimuthal oscillations. However, results presented here show a more spread oscillation due to heat conduction, which causes a smoother temperature profile and a wider ionization region.

\section{Parametric variations}

This paragraph presents the results of the azimuthal global stability analysis for the background solutions presented in Table II. This analysis consists in the search of self-excited oscillations in the frequency space for the different background solutions under evaluation and for different wave mode numbers. For comparison purposes, the unstable solution presented in the previous paragraph is used as reference for this analysis.

Here, it is interesting to check whether the main trends observed experimentally are also reproduced with the linear 2D time-dependent model, in particular, with respect to the variation of the oscillation frequency. Moreover, it is also interesting to analyze mode numbers $m>1$ as well since experiments show that those modes become dominant in the case of large thrusters. ${ }^{13}$

Note that the frequency of the oscillation is expected to grow linearly with the mode number. This does not mean that the oscillation travels faster since, for example, for the same phase speed, an $m=2$ oscillation has a frequency twice as large as an $m=1$ oscillation. In fact, the phase speed of the azimuthal oscillation, $v_{y}$, is given in terms of the oscillation frequency, $f$, and the wave mode number, $m$, as

$$
v_{y}=-\frac{2 \pi R_{m} f}{m} .
$$

Equation (3) corresponds to the usual definition of the phase velocity, $v_{y}=\omega / k_{y}$, where $\omega$ is the angular frequency and $k_{y}$ is the wave number. In Eq. (3), the angular frequency is expressed as $\omega=2 \pi f$ and the wave number is expressed as $k_{y}=-m / R_{m}$.

It is important to mention that the value of the frequency observed for the reference background solution $(f=24.9 \mathrm{kHz})$ presented in the previous paragraph is in the upper-side of the range seen in experiments ${ }^{2,13}(f \approx 5-25 \mathrm{kHz})$, but still within the modelling error margins of the linear 2D time-dependent model. This mild discrepancy between the experiments and the frequency of the $m=1$ oscillation causes that for higher wave mode numbers, the discrepancy becomes larger for the oscillation frequency in absolute terms. Note in any case that in this study we are seeking qualitative rather than quantitative agreement in order to characterize physically the spoke. Therefore, this discrepancy is not considered critical for the purposes of this study.

Table II shows the variation of the frequency of the unstable oscillation found for different background solutions and wave numbers.

The following trends are observed:

- As the discharge voltage is increased, or the mass flow rate is decreased, the frequency of the azimuthal oscillation normally increases linearly. Those trends are similar to those of the breathing mode, showing a similarity between that axial oscillation and the azimuthal spoke oscillation. These features may indicate a relation between the frequency of the oscillations and the position of the ionization region in the background solution. The ionization region moves downstream when the discharge voltage increases or the mass flow decreases, ${ }^{37}$ and at the same time, the oscillation frequency of the breathing mode may be related approximately to the distance from the ionization region to the anode using a basic predator-prey model. ${ }^{48}$

- As mentioned above, the frequency of the oscillation increases linearly with the wave mode number. Additionally, depending on the background case, the mode number with the highest growth rate may be $m=1, m=2$, or $m=3$, although as a general trend, the higher the mode number is, the less unstable solutions exist. Modes $m>1$ seem to become dominant for higher mass flow rates and larger channels since the growth rate is higher in those cases, although, this trend is not as evident as the previous ones.

- Even though not shown in Table II, no unstable solution exists travelling in $-\mathbf{E} \times \mathbf{B}$ direction $(m=-1)$, indicating that the oscillation detected in the simulations has a clear preference to travel in the $+\mathbf{E} \times \mathbf{B}$ direction, as normally found in experiments.

Apart from the trends mentioned above, the simulations show oscillations with some distinct features, corresponding 

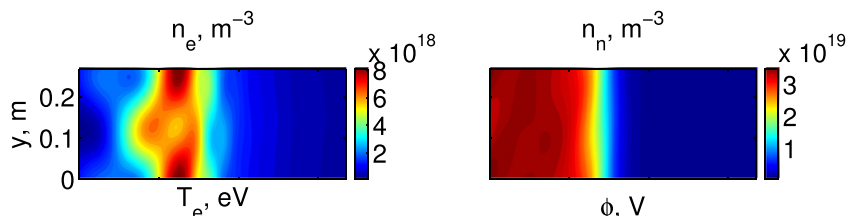

$\phi, \mathrm{V}$
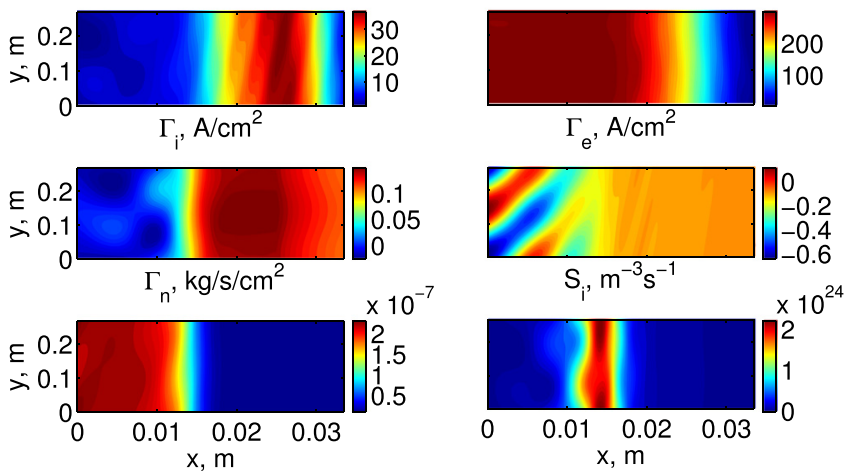

FIG. 6. Oscillations of the main macroscopic variables as combinations of the background solution and the perturbations shown as functions of $x$ and $y$ at $t=0$ for an $m=1$ self-excited oscillation of the perturbation problem for the reference case IId. Variables shown (from left to right and upper to lower): plasma density, $n_{e}$; neutral density, $n_{n}$; electron temperature, $T_{e}$; electric potential, $\phi$; ion axial flux, $\Gamma_{i}$; electron axial flux, $\Gamma_{e}$; neutral axial flux, $\Gamma_{n}$; and ionization source term, $S_{i}=n \nu_{i}$.

to those cases marked with an asterisk $(*)$ in Table II, namely, cases IId and IIe with $m=1$ of the parametric variation on the mass flow. Figs. 6 and 7 show the $m=1$ oscillation for case IId. The differences with respect to the oscillation of the reference background solution shown in Figs. 3-5 allow a clearer understanding of the physics behind. The neutral density follows a similar pattern to that of the breathing mode, and the tilt angle of the oscillation is close to zero. It is believed that the mechanism behind all oscillations in Table II is the same, contrary to our first provisional conclusion, ${ }^{42}$ where cases IId and IIe were considered of different nature. In order to justify this further, in
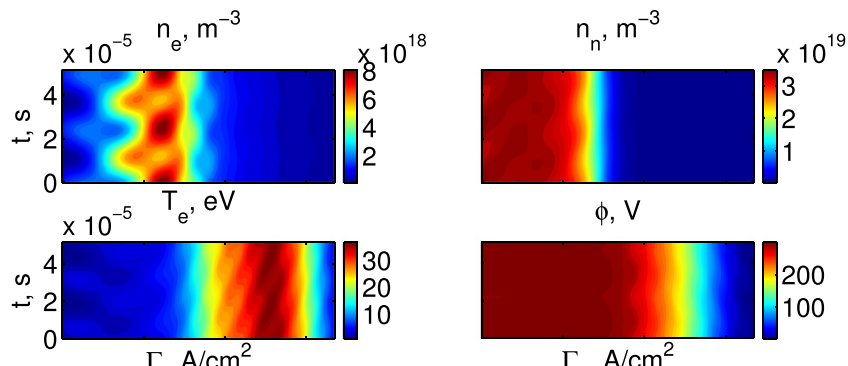

$\phi, \mathrm{V}$

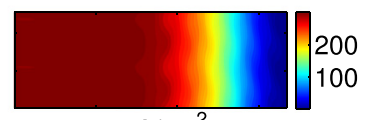

$\Gamma_{e}, \mathrm{~A} / \mathrm{cm}^{2}$
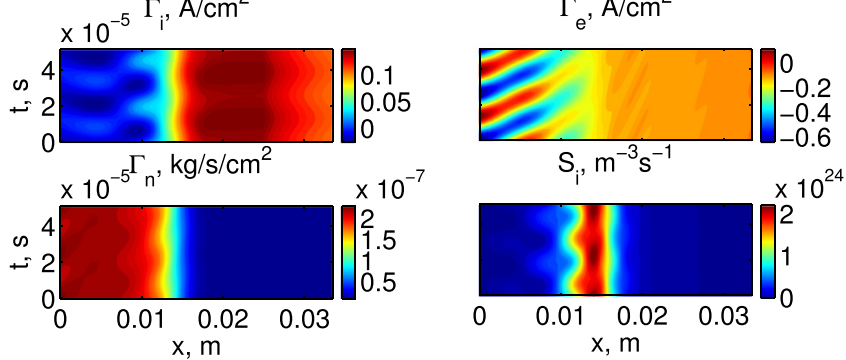

FIG. 7. Oscillations of the main macroscopic variables as combinations of the background solution and the perturbations shown as functions of $x$ and $t$ at $y=0$ for an $m=1$ self-excited oscillation of the perturbation problem for the reference case IId. The variables shown are the same of Fig. 6.
Section IV, an analysis is carried out with simplified perturbation models showing that the oscillation detected in cases IId and IIe is indeed similar in nature to the one shown in Figs. 3-5.

According to Figs. 6 and 7, the oscillation is characterized by the presence in the azimuthal direction of several ionization fronts that move back and forth with different phases, causing a plasma density variation near the anode that rotates in the $\mathbf{E} \times \mathbf{B}$ direction. This structure resembles the standing wave of plasma density in the breathing mode, even if in the latter there is no rotation in the azimuthal direction. In fact, the possibility of a localized breathing mode rotating in the azimuthal direction has already been proposed previously by the authors, ${ }^{43}$ based on a theoretical analysis, and by Sekerak et al., ${ }^{17}$ based on experimental results.

Aside from the parametric variations presented in the previous paragraphs, it is also interesting to analyze the possible influence of the Bohm diffusion present in the background solution on the simulated spoke. One of the possible drivers for the anomalous diffusion in Hall thrusters is an enhanced electron transport caused by correlated azimuthal oscillations of electric field and plasma density. And, in fact, the azimuthal oscillations observed in this study may indeed participate in the anomalous diffusion as shown recently. ${ }^{43}$ However, in order to have consistent results between the zero-th and first order solutions, the Bohm diffusion used in the zero-th order problem should come from the first order problem.

To analyze this and check the consistency of the results shown above, another parametric variation is carried out with the goal of decreasing as much as possible the Bohm diffusion in the background solution, whose stability can then be evaluated using the 2D linear time-dependent model. As part of this parametric variation, the Bohm diffusion coefficient is reduced five times, from the value in Table I, i.e., from $\alpha_{B} \sim 0.01$ to $\alpha_{B} \sim 0.002$. And even in this reduceddiffusion background scenario, the stability analysis shows that there exists an unstable oscillation with a frequency of $f=48.7 \mathrm{kHz}$, with similar properties to the oscillation observed for the reference state. This fact proves that the observed oscillation is not a result of the empirical Bohm diffusion added to the background solution but comes naturally from the Hall discharge physics.

\section{ANALYSIS OF SIMPLIFIED MODELS}

In Sections II and III, the complete linear 2D timedependent model has been presented and used. However, provided that the zero-th and first order solutions are obtained separately, it is possible to consider different terms and equations in the background and perturbation problems. While all terms must be kept in the zero-th order problem in order to maintain the background solution similar to experimental results, some terms may be safely neglected in the perturbation problem based on the relative size of the perturbations with respect to the background state for a given oscillation. This allows simplifying the formulation of the first-order problem and keeping only the terms in the perturbation model that are strictly 
necessary to give rise to the observed oscillations. Obviously, this approach can only be applied in theoretical and numerical analyses, as in real plasmas in Hall thrusters all variables are perturbed. However, this method allows studying more in detail the mechanism of the oscillation.

Let us consider the azimuthal oscillation shown in Figs. $3-5$. In that oscillation, the relative size of the neutral density perturbation is orders of magnitude smaller than that of plasma density. This gives the hint to remove from the perturbation model the continuity and momentum equations of the neutral species and set to zero the perturbations of neutral density and velocity, and ionization, wall recombination, and wall losses. Furthermore, the azimuthal ion velocity perturbation is also negligible in relative terms compared to other perturbations and thus may also be neglected. If those terms and equations are removed from the perturbation problem, a simplified model is obtained. With this simplified model, an $m=1$ unstable oscillation is still found for the reference background state with a frequency of $f=44.8 \mathrm{kHz}$, slightly higher than with the full model. Fig. 8 shows for that oscillation the contour maps in the $x-y$ space of the main macroscopic variables. The similarity between Figs. 8 and 3 is an indication that the neglected perturbations are indeed not relevant for this azimuthal oscillation.

If we now consider the azimuthal oscillations of cases IId and IIe shown in Figs. 6 and 7, the same procedure may be carried out. And again, self-excited oscillations with a mode number $m=1$ are also found with frequencies $f=38.0$ $\mathrm{kHz}$ and $f=13.4 \mathrm{kHz}$, respectively. The same pattern of localized breathing mode oscillations observed in Fig. 6 is found, what validates the simplification carried out.

In case the simplified model described above is applied to the case of the breathing mode $(m=0)$, no unstable oscillations are found. This is expected as it is well known that the breathing mode is characterized by the depletion and
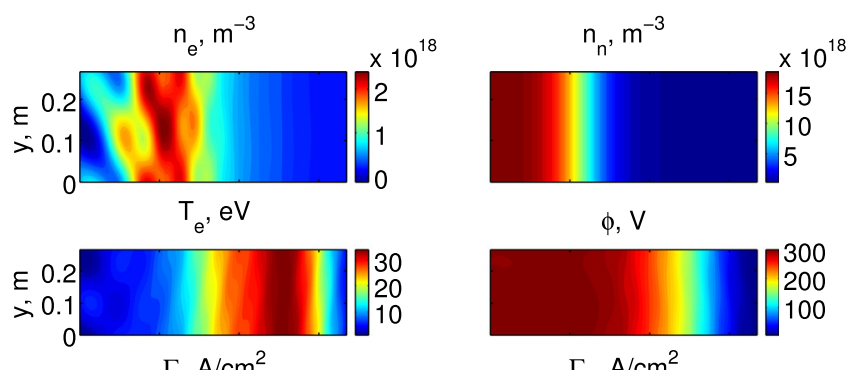

$\Gamma_{i}, \mathrm{~A} / \mathrm{cm}^{2}$
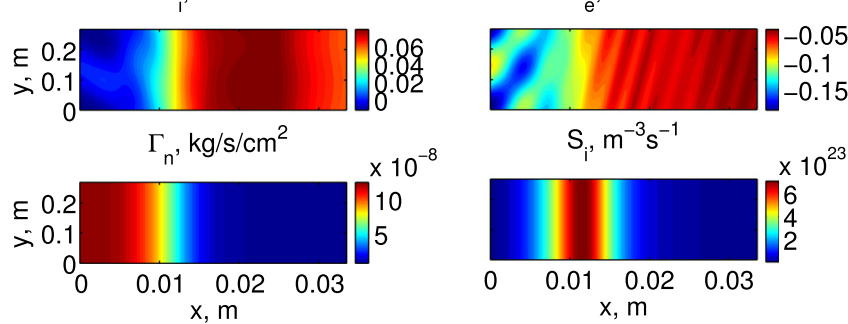

FIG. 8. Oscillations of the main macroscopic variables as combinations of the background solution and the perturbations shown as functions of $x$ and $y$ at $t=0$ for an $m=1$ self-excited oscillation of the perturbation problem for the reference case from Fig. 1 and the perturbation model excluding neutral equations, ionization, wall recombination, wall losses, and azimuthal ion velocity. The variables shown are the same of Fig. 6. replenishment of neutral gas in the ionization region. Thus, if no neutral equations are included in the simplified perturbation model, it is expected that no self-modes appear for the case $m=0$.

If the simplification of the perturbation model is taken one step further and heat conduction terms are removed as well, similar azimuthal unstable solutions are also found. However, if perturbations of additional macroscopic variables are also removed from the perturbation model, such as electron temperature, ion axial velocity, or plasma density, unstable oscillations are no longer detected.

So far, the largest set of simplifications that still allow reproducing unstable azimuthal oscillations yields a model consisting only of continuity and momentum equations for electrons and ions without ionization and wall recombination, together with the electron energy equation without heat conduction and wall losses.

Another element that can promote the appearance of these azimuthal oscillations is the conditions at the limits of the simulation domain. In fact, if in the simplified model just described the linearised Bohm condition is relaxed such that the axial ion velocity perturbation is zero at the anode, then the unstable oscillations no longer appear. This result indicates that the azimuthal oscillations are promoted by the coupling of the perturbations of electron temperature and ion axial velocity in the anode plasma-sheath transition. However, in case the Bohm condition is relaxed in the original full perturbation model, azimuthal oscillations are still detected. It is only when neutral equations and ionization terms are excluded from the perturbation model, together with the relaxation of the Bohm condition at the anode, that the unstable azimuthal oscillations disappear.

Based on the previous results, it is possible to conclude that the azimuthal oscillations observed in the simulations are due to both the ionization process and the coupling of the perturbations of electron temperature and ion velocity at the anode sheath transition due to the Bohm condition. The latter element can only be included in global stability analysis through the boundary conditions of the first-order problem, while local methods cannot account for such effects.

Another result from this analysis is that the oscillations observed in the reference case and in cases IId and IIe behave similarly with respect to the use of simplified models. Thus, it seems that, even if part of the features of those oscillations are different, the mechanism behind them is the same.

Note that the conclusions presented here do not confirm those previously reported by the authors, ${ }^{42}$ where heat conduction effects were considered by the authors as a possible cause of azimuthal oscillations. After careful re-examination, the new results indicate that heat conduction plays no role in the azimuthal oscillations.

\section{GLOBAL VS. LOCAL STABILITY ANALYSES}

Another interesting analysis that can be performed with the aid of simplified perturbation models is the comparison between local and global stability analyses. Both methods start from a given set of non-linear governing equations, as 
for instance equations in (1), and apply the small perturbations hypothesis to linearise the model around a given background state. In global analyses, the perturbations are then assumed to be of the Fourier form given in Eq. (2). That type of Fourier form is the basis of global stability studies, where the resulting formulation is not algebraic but consists of differential equations to solve for $\bar{f}(x)$. Inhomogeneities in $x$ are accounted for in these global methods, and therefore, within the linear regime, the global approach is fully consistent with the inhomogeneous plasmas found in Hall thrusters.

Contrary to global methods, in local analyses, the axial variation of $\bar{f}(x)$ in Eq. (2) is also expressed in Fourier form resulting in

$$
\hat{f}(t, x, y)=\bar{f} \exp \left(i k_{x} x\right) \exp \left(-i \omega t+i k_{y} y\right),
$$

where $k_{x}$ is the axial wave number and $\bar{f}$ is the local coefficient of the Fourier expression at a fixed $x_{0}$.

Once applied to the governing equations, the local method yields an algebraic local problem that is independent for each axial coordinate, $x_{0}$. Thus, in these local analyses, it is necessary to freeze the zero-th order variables and their gradients and consider them constant, even if in reality the plasma is inhomogeneous along $x$, so that the Fourier analysis can be carried out in the $x$ direction. This approach can be consistent when the length scale of the axial variation of the zero-th order variables $\left(L_{x}\right)$ is much larger than the axial wave-length of the perturbations $\left(k_{x} L_{x} \gg 1\right)$. This is the socalled Boussinesque approximation ${ }^{25}$ and allows reducing the formulation to an algebraic local problem, as opposed to the differential problem obtained in global methods. In Hall thrusters, that condition is not met and thus solutions from local stability analyses must be considered as approximate solutions with limited consistency.

Many local linear stability analyses, ,20-29 including the first paper of this series, ${ }^{1}$ have been carried out so far by the Hall thruster community in an attempt to better understand the mechanism behind azimuthal oscillations inherent in Hall thrusters. However, as described above, in the case of Hall thrusters, given the inhomogeneities along the channel, local analyses are not completely consistent as they neglect the axial variation of the macroscopic plasma variables. Some authors claim that local stability analyses can even yield different stability criteria depending on how the equations are linearised. ${ }^{47}$ Furthermore, boundary conditions, such as mass flow rate or discharge voltage, which can also be the driver for some instabilities, cannot be taken into account in local methods, while global methods consider them consistently as linearised boundary conditions in the resulting system of ordinary differential equations.

In order to illustrate and discuss the differences between the results from local and global analyses, the stability criterion proposed by Esipchuk and Tilinin ${ }^{21}$ has been evaluated throughout given axial profiles of the Hall discharge looking for oscillations with a wave mode number $m=1$. That stability criterion is based on electrostatic two-fluid equations of ions and electrons suited for the low-frequency regime. Frias et $a l^{25}$ have revisited the same local stability problem, obtaining a dispersion relation qualitatively similar in form, but quantitatively different to that of Esipchuk and Tilinin due to the inclusion of the complete electron compressibility. That improved dispersion relation has been used by Frias et al. in a separate publication ${ }^{26}$ to evaluate the local stability at each axial location of the profiles of three different Hall thrusters.

The upper plot of Fig. 9 shows the results obtained with the local stability criterion of Esipchuk and Tilinin for the reference background solution shown in Fig. 1. Two unstable areas are identified, one in the ionization region near the anode and another one in the plume, where the axial gradient of the magnetic field is negative. Similarly, as shown in the lower plot of Fig. 9, the results obtained with the stability criterion proposed by Frias et al. ${ }^{25}$ also show two regions of unstable azimuthal oscillations, in line with the results obtained by Frias et $a l .^{26}$ for other Hall discharge profiles. Finally, it is worth to point out that similar results are obtained for the different background profiles in Table II although this fact is not shown in any figure.

In order to perform the comparison between local and global analyses, the perturbation equations of the linearised 2D time-dependent model presented above are simplified to keep only the same equations and terms of the local model of Esipchuk and Tilinin. This translates into retaining only ion and electron continuity and momentum equations in the simplified perturbation model, with which the global stability analysis can then be performed. Self-excited solutions observed with that simplified model would then correspond
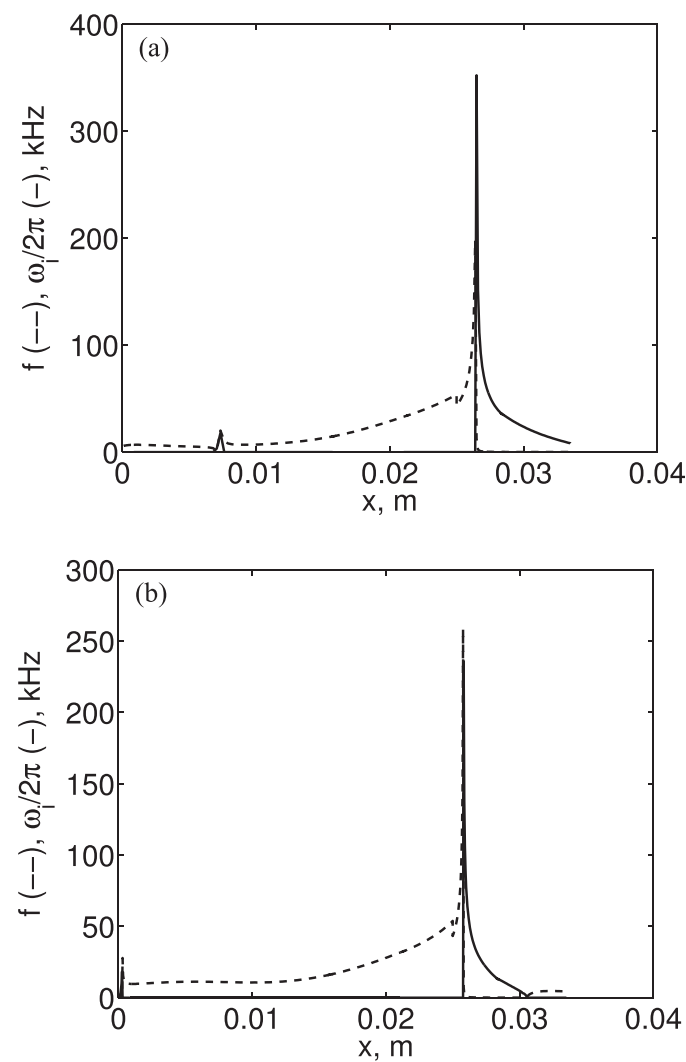

FIG. 9. Growth rate (continuous line) and frequency (dashed line) in $\mathrm{kHz}$ for the $m=1$ oscillations predicted with the local stability criterion proposed by Esipchuk and Tilinin ${ }^{21}$ (top) and by Frias et al. ${ }^{25}$ (bottom) evaluated at each axial location of background solution corresponding to Fig. 1. 


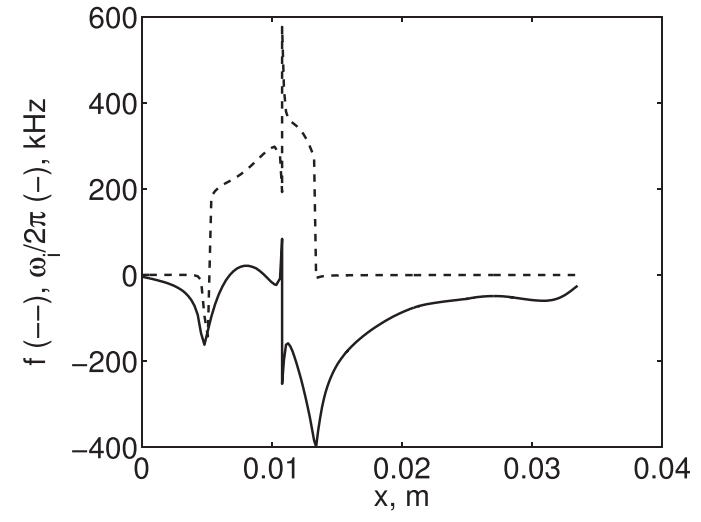

FIG. 10. Growth rate (continuous line) and frequency (dashed line) in $\mathrm{kHz}$ for the $m=1$ oscillations predicted with the local stability criterion proposed by Escobar and Ahedo ${ }^{1}$ evaluated at each axial location of background solution corresponding to Fig. 1.

to those predicted by the local stability criterion. However, the results obtained indicate that no unstable solutions are found for any of the profiles in Table II, where the local analysis predicts unstable oscillations. This seems to indicate that, as already anticipated by some authors, ${ }^{41}$ results from local stability analyses of the Hall Thruster discharge must be analysed with care.

However, the dispersion relation from the local stability analysis presented in the first paper of this series ${ }^{1}$ can also be applied to the profile of the reference background solution. That local stability analysis, aside from continuity, momentum, and energy equations for ions and electrons, includes also conservation equations for the neutral species and takes into account the ionization process via various source terms. Therefore, it is consistent with the $2 \mathrm{D}$ linear time-dependent model used in this article. The results of this analysis are shown in Fig. 10, where the large peak observed is associated with the condition $d n_{0} / d x=0$, which is a singularity for the local dispersion relation used. In that figure, it is possible to observe that for this reference case, there is an area of local instabilities $\left(\omega_{i}>0\right)$ in the ionization region. Although not shown in any figure, other background solutions in Table II show similar local instabilities. This fact is in line with the conclusions derived by the authors in Part I of this study, ${ }^{1}$ where a local instability is predicted in the part of the ionization region, where $v_{i x 0}>0, d v_{i x 0} / d x>0$, and $d n_{0} /$ $d x>0$. Moreover, those local instabilities in the ionization region could match the results of the global analysis presented in this article. However, it is not straightforward to establish a direct link between the local and global methods since, according to the results of this study, the Bohm condition at the anode plasma-sheath transition also plays a key role in the promotion of these low frequency oscillations, and that condition cannot be considered in local stability analyses. ${ }^{1}$

\section{CONCLUSIONS}

A linearised 2D time-dependent model has been used for the analysis of the stability of the Hall discharge in the azimuthal direction and the detection of azimuthal oscillations in the low frequency range. The 2D model builds on the 1D model of Ahedo et al. and has been shown to be capable of reproducing breathing mode oscillations in the axial direction. Spoke oscillations have been also detected in the ionization region rotating in the $+\mathbf{E} \times \mathbf{B}$ direction. Additionally, properties similar to those observed experimentally have been revealed with various parametric investigations on discharge voltage, mass flow, and thruster width.

Simplified perturbation models have been used to analyze the physical mechanism behind the oscillations. Results seem to indicate that the observed azimuthal oscillations are linked to oscillations of the ionization process and to the Bohm condition in the transition to the anode sheath. The latter is a feature that can only be considered with global methods as it enters the model through the boundary conditions of the perturbation problem.

Additionally, a comparison between global and local stability analyses has also been carried out showing discrepancies between both methods. The results of this comparison indicate that oscillations detected with the local models of Esipchuk and Tilinin and of Frias et al. do not show up in the global analysis, while those predicted by the authors with a different local model ${ }^{1}$ might be somehow related to the ones observed in this study with the global approach.

\section{ACKNOWLEDGMENTS}

Support to D. Escobar has been provided by the Air Force Office of Scientific Research, Air Force Material Command, USAF, under Grant No. FA8655-13-1-3033. Support to E. Ahedo has come from Spain's R\&D National Plan (Project ESP-2013-41052).

${ }^{1}$ D. Escobar and E. Ahedo, "Low frequency azimuthal stability of the ionization region of the Hall thruster discharge. I. Local analysis," Phys. Plasmas 21(4), 043505 (2014)

${ }^{2}$ E. Y. Choueiri, "Plasma oscillations in Hall thrusters," Phys. Plasmas 8, 1411 (2001).

${ }^{3}$ G. S. Janes and R. S. Lowder, "Anomalous electron diffusion and ion acceleration in a low-density plasma,” Phys. Fluids 9, 1115 (1966).

${ }^{4}$ Y. B. Esipchuk, A. I. Morozov, G. N. Tilinin, and A. V. Trofimov, "Plasma oscillations in closed-drift accelerators with an extended acceleration zone," Sov. Phys.-Tech. Phys. 18(7), 928-932 (1974).

${ }^{5}$ P. J. Lomas and J. D. Kilkenny, "Electrothermal instabilities in a Hall accelerator," Plasma Phys. 19, 329 (1977).

${ }^{6}$ Y. Raitses, A. Smirnov, and N. J. Fisch, "Effects of enhanced cathode electron emission on Hall thruster operation," Phys. Plasmas 16, 057106 (2009).

${ }^{7}$ Y. Raitses, M. Griswold, L. Ellison, J. Parker, and N. J. Fisch, "Studies of rotating spoke oscillations in cylindrical Hall thrusters," AIAA Paper 2012-4179, 2012.

${ }^{8}$ J. B. Parker, Y. Raitses, and N. J. Fisch, "Transition in electron transport in a cylindrical Hall thruster,” Appl. Phys. Lett. 97, 091501 (2010).

${ }^{9}$ C. L. Ellison, Y. Raitses, and N. J. Fisch, "Direct measurement of spokeinduced, cross-field electron current in a cylindrical Hall thruster," in Proceedings of the 32nd International Electric Propulsion Conference (2011), IEPC-2011-173.

${ }^{10}$ C. L. Ellison, Y. Raitses, and N. J. Fisch, "Cross-field electron transport induced by a rotating spoke in a cylindrical Hall thruster," Phys. Plasmas 19, 013503 (2012).

${ }^{11}$ Y. Shi, Y. Raitses, and A. Diallo, "Driving low frequency azimuthal mode in cylindrical hall thruster with a segmented anode," in Proceedings of the 33rd International Electric Propulsion Conference (2013), IEPC-2013176.

${ }^{12}$ M. S. McDonald, C. K. Bellant, B. A. S. Pierre, and A. D. Gallimore, "Measurement of cross-field electron current in a Hall thruster due to rotating spoke instabilities," AIAA Paper 2011-5810, 2011. 
${ }^{13}$ M. S. McDonald and A. D. Gallimore, "Parametric investigation of the rotating spoke instability in Hall thrusters," in Proceedings of the 32nd International Electric Propulsion Conference (2011), IEPC-2011-242.

${ }^{14}$ M. S. McDonald and A. D. Gallimore, "Rotating spoke instabilities in Hall thrusters,” IEEE Trans. Plasma Sci. 39(11), 2952-2953 (2011).

${ }^{15}$ M. S. McDonald and A. D. Gallimore, "Comparison of breathing and spoke mode strength in the H6 Hall thruster using high speed imaging," in Proceedings of the 33rd International Electric Propulsion Conference (2013), IEPC-2013-353.

${ }^{16}$ M. Sekerak, M. McDonald, R. Hofer, and A. Gallimore, "Hall thruster plume measurements from high-speed dual Langmuir probes with ion saturation reference," in 2013 IEEE Aerospace Conference (IEEE, 2013), pp. $1-16$.

${ }^{17}$ M. Sekerak, B. Longmier, A. D. Gallimore, D. Brown, R. Hofer, and J. Polk, "Azimuthal spoke propagation in Hall effect thrusters," in Proceedings of the 33rd International Electric Propulsion Conference (2013), IEPC-2013-143.

${ }^{18}$ D. Liu, "Two-dimensional time-dependent plasma structures of a Halleffect thruster," Ph.D. thesis (Department of Aeronautics and Astronautics, Graduate School of Engineering and Management, Air Force Institute of Technology, 2011).

${ }^{19}$ D. Liu, R. E. Huffman, R. D. Branam, and W. A. Hargus, "Ultrahighspeed imaging of Hall-thruster discharge oscillations with krypton propellant," IEEE Trans. Plasma Sci. 39(11), 2926-2927 (2011).

${ }^{20}$ A. I. Morozov, Y. V. Esipchuk, and A. M. Kapulkin, "Effect of the magnetic field on a closed electron drift accelerator," Sov. Phys.-Tech. Phys. 17(3), 482-487 (1972).

${ }^{21}$ Y. B. Esipchuk and G. N. Tilinin, "Drift instability in a Hall-current plasma accelerator," Sov. Phys.-Tech. Phys. 21(4), 417-423 (1976).

${ }^{22}$ A. Kapulkin and M. Guelman, "Low frequency instability and enhanced transfer of electrons in near-anode region of Hall thruster," in Proceedings of the 30th International Electric Propulsion Conference (2007), IEPC2007-079.

${ }^{23}$ A. Kapulkin and M. M. Guelman, "Low-frequency instability in nearanode region of Hall thruster," IEEE Trans. Plasma Sci. 36(5), 2082-2087 (2008).

${ }^{24}$ A. I. Smolyakov, W. Frias, Y. Raitses, and N. J. Fisch, "Gradient instabilities in Hall thruster plasmas," in Proceedings of the 32 nd International Electric Propulsion Conference (2011), IEPC-2011-271.

${ }^{25}$ W. Frias, A. I. Smolyakov, and I. D. Kaganovich, "Long wavelength gradient drift instability in Hall plasma devices. I. Fluid theory," Phys. Plasmas 19, 072112 (2012).

${ }^{26}$ W. Frias, A. I. Smolyakov, I. D. Kaganovich, and Y. Raitses, "Long wavelength gradient drift instability in Hall plasma devices. II. Applications," Phys. Plasmas 20, 052108 (2013).

${ }^{27}$ E. Chesta, N. B. Meezan, and M. A. Cappelli, "Stability of a magnetized Hall plasma discharge,” J. Appl. Phys. 89(6), 3099-3107 (2001).

${ }^{28}$ J. M. Gallardo and E. Ahedo, "On the anomalous diffusion mechanism in Hall-effect thrusters," in Proceedings of 29th International Electric Propulsion Conference (2005), IEPC-2005-117.

${ }^{29}$ H. K. Malik and S. Singh, "Resistive instability in a Hall plasma discharge under ionization effect,” Phys. Plasmas 20, 052115 (2013).
${ }^{30}$ A. A. Litvak and N. J. Fisch, "Rayleigh instability in Hall thrusters," Phys. Plasmas 11, 1379 (2004).

${ }^{31}$ A. M. Kapulkin and V. F. Prisnyakov, "Dissipative method of suppression of electron drift instability in SPT," in Proceedings of the 24th International Electric Propulsion Conference (1995), IEPC-95-37.

${ }^{32}$ A. Kapulkin, J. Ashkenazy, A. Kogan, G. Appelbaum, D. Alkalay, and M. Guelman, "Electron instabilities in Hall thrusters: modelling and application to electric field diagnostics," in Proceedings of the 28th International Electric Propulsion Conference (2003), IEPC-2003-100.

${ }^{33}$ A. Kapulkin and M. Guelman, "Lower-hybrid instability in Hall thruster," in Proceedings of the 29th International Electric Propulsion Conference (2005), IEPC-2005-88.

${ }^{34}$ E. Ahedo, J. M. Gallardo, and M. Martinez-Sanchez, "Effects of the radial plasma-wall interaction on the Hall thruster discharge," Phys. Plasmas 10, 3397 (2003).

${ }^{35}$ E. Ahedo, J. M. Gallardo, and M. Martinez-Sanchez, "Model of the plasma discharge in a Hall thruster with heat conduction," Phys. Plasmas 9, 4061 (2002).

${ }^{36}$ E. Ahedo, P. Martinez, and M. Martinez-Sanchez, "One-dimensional model of the plasma flow in a Hall thruster," Phys. Plasmas 8, 3058 (2001).

${ }^{37}$ E. Ahedo and D. Escobar, "Influence of design and operation parameters on Hall thruster performances," J. Appl. Phys. 96, 983 (2004).

${ }^{38}$ E. Ahedo and F. I. Parra, "A model of the two-stage Hall thruster discharge,” J. Appl. Phys. 98, 023303 (2005).

${ }^{39}$ E. Ahedo, P. Martinez, and M. Martinez-Sanchez, "Steady and linearlyunsteady analysis of a Hall thruster with an internal sonic point," AIAA Paper 2000-3655, 2000.

${ }^{40}$ R. Noguchi, M. Martinez-Sanchez, and E. Ahedo, "Linear 1-d analysis of oscillations in Hall thrusters," in Proceedings of the 26th International Electric Propulsion Conference (1999), IEPC-99-105.

${ }^{41}$ S. Barral, V. Lapuerta, A. Sancho, and E. Ahedo, "Numerical investigation of low-frequency longitudinal oscillations in Hall thrusters," in Proceedings of the 29th International Electric Propulsion Conference (2005), IEPC-2005-120.

${ }^{42} \mathrm{D}$. Escobar and E. Ahedo, "Analysing the azimuthal spoke oscillation of Hall thrusters via numerical simulation," in Proceedings of the 50th Joint Propulsion Conference (2014), IEPC-2014-3512.

${ }^{43}$ D. Escobar and E. Ahedo, "Global stability analysis of azimuthal oscillations in Hall thrusters," IEEE Trans. Plasma Sci. 43(1), 149 (2015).

${ }^{44}$ J. P. Boeuf and L. Garrigues, "Low frequency oscillations in a stationary plasma thruster," J. Appl. Phys. 84, 3541 (1998).

${ }^{45} \mathrm{~S}$. Barral and E. Ahedo, "Theoretical study of the breathing mode in Hall thrusters," AIAA Paper 2006-5172, 2006.

${ }^{46} \mathrm{~S}$. Barral and E. Ahedo, "Low-frequency model of breathing oscillations in Hall discharges,” Phys. Rev. E 79(4), 046401 (2009).

${ }^{47}$ S. Barral, K. Makowski, Z. Peradzyński, and M. Dudeck, “Transit-time instability in Hall thrusters," Phys. Plasmas 12, 073504 (2005).

${ }^{48}$ J. M. Fife, "Hybrid-PIC modelling and electrostatic probe survey of Hall thrusters," Ph.D. thesis (Massachusetts Institute of Technology, 1998). 